\title{
VIOLÊNCIA CONTRA A PESSOA IDOSA EM TEMPO DE PANDEMIA DA COVID-19
}

\author{
Elizabeth Moura Soares de Souza' \\ ORCID: 0000-0002-5889-8197 \\ Fabia Maria de Lima" \\ ORCID: 0000-0001-9992-6559
}

Jack Roberto Silva Fhon"'I

ORCID: 0000-0002-1880-4379

Daiane de Souza Fernandesiv

ORCID: 0000-0001-6629-4222

Deuzany Bezerra de Melo Leãov

ORCID: 0000-0002-5510-0733

Anna Karla de Oliveira Tito Borb ${ }^{\text {vi }}$ ORCID: 0000-0002-9385-6806

Universidade Federal de Alagoas. Maceió, Alagoas, Brasil.

"Universidade de Pernambuco. Recife, Pernambuco, Brasil.

"' Universidade de São Paulo. São Paulo, São Paulo, Brasil.

Iv Universidade Federal do Pará. Belém, Pará, Brasil.

"Universidade de Pernambuco. Recife, Pernambuco, Brasil.

VII Universidade Federal de Pernambuco. Recife, Pernambuco, Brasil.

Autor Correspondente:

Elizabeth Moura Soares de Souza

E-mail:elmososo@gmail.com

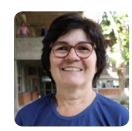

Como citar:

Souza EMS, Lima FM, Fhon JRS, Fernandes DS, Leão DBM, Borb AKO. Violência contra a pessoa idosa em tempo de pandemia da covid-19. In: Santana RF (Org.). Enfermagem gerontologica no cuidado do idoso em tempos da COVID 19. 2.ed.rev. Brasilia, DF: Editora ABEn; 2020. p 144-149. (Serie Enfermagem e Pandemias, 2). https://doi.org/10.51234/aben.20.e02.c22

\section{INTRODUÇÃO}

A infecção aguda do trato respiratório conhecida com casos de pneumonia ocasionada pelo novo coronavírus iniciou-se em Wuhan, China, em dezembro de 2019 sendo declarada como pandemia em 11 de março de 2020(1). Dados obtidos até 11 de junho de 2020 sobre a pandemia no site da Johns Hopkins indica 7.403.713 casos confirmados sendo que Estados Unidos, Brasil e Rússia encontram-se no topo da lista. Quanto aos óbitos, o site informa que houve 417.174 casos sendo Estados Unidos, Reino Unido e Brasil que apresentam o maior número de mortes ${ }^{(2)}$.

A infecção é transmitida através de grandes gotículas geradas durante a tosse e espirros por pacientes sintomáticos, mas também pode ocorrer em pessoas assintomáticas e antes do início dos sintomas. Ademais, todas as idades são susceptíveis a adoecer em especial o idoso(3).

Com o início da pandemia, a Organização Mundial da Saúde (OMS) indicou algumas recomendações sendo uma delas o isolamento e/ou afastamento social, em especial nas populações vulneráveis, entre elas, os idosos. No entanto, é sabido que o isolamento entre idosos causa preocupação devido ao seu risco aumentado de problemas de saúde física, neurocognitivos, depressão, ansiedade ${ }^{(4)}$ e aumento do risco de violência(5).

A OMS define violência como: "o uso intencional de força ou poder físico, ameaçado ou real, contra si mesmo, outra pessoa ou contra um grupo ou comunidade, que resulte ou tenha uma alta probabilidade de resultar em ferimentos, morte, dano psicológico, desenvolvimento inadequado ou privação" (WHO, 2020) e pode ser de causa física, psicológica, sexual, econômica, negligência, autonegligência e abandono ${ }^{(6)}$.

Apesar da importância do isolamento social para conter o avanço da disseminação do vírus com a tentativa de proteger os mais vulneráveis, entre eles os idosos, população de maior risco para sofrer violência, uma vez que os membros das 
famílias, muitas vezes, já eram agressores e com o isolamento de todos, o estresse aumenta entre as pessoas em decorrência da diminuição da renda, espaço inadequado para abrigar todos da família, conflitos diários, assim, o idoso, considerado mais frágil passa a ser um membro familiar oprimido pelos seus familiares.

Essa condição de maior vulnerabilidade do idoso deve merecer atenção especial da sociedade. Diante da necessidade de refletir sobre o assunto em tempos de pandemia à luz da literatura atual, o cuidado de enfermagem ao idoso deve ser de atenção redobrado, nesse momento de mudanças sociais.

\section{OBJETIVO}

Refletir sobre a violência contra a pessoa idosa no domicílio durante a pandemia da COVID-19.

\section{MÉTODOS}

Trata-se de um levantamento na literatura com a estruturação de uma revisão narrativa sobre a violência contra as pessoas idosas em isolamento no domicílio durante a pandemia da COVID-19. As reflexões foram embasadas à luz de artigos internacionais e nacionais relacionados ao tema. As observações identificadas nos auxiliaram a uma análise teórico-científica, pois a violência contra o idoso apresenta-se como um fenômeno multifatorial, dotada de complexidade.

Para análise da temática "violência contra as pessoas idosas em isolamento no domicílio durante a pandemia do COVID-19" na busca de artigos internacional utilizamos na Medline (via Pubmed) os termos MeSH: "pandemics", "coronavirus", "violencia" and "elderly"; e nacional buscamos na Biblioteca Virtual em Saúde (BVS) as palavras chaves do DESC: "pandemia", "coronavírus", "violencia" e "idoso", com operador booleano "AND". O período da pesquisa foi de maio a junho/2020 e realizado a seleção de acordo com a necessidade de construção da temática.

\section{REFLEXÕES}

A violência caracteriza-se por um ato antissocial humano, sem formas, sem limites, muitas das vezes impiedosa e desumana. Por essa razão, a violência pode adotar várias configurações e passar por diferentes cenários, sendo impossível dimensioná-la em toda a sua abrangência.

De acordo com o modelo ecológico proposto pela Organização Mundial da Saúde (OMS), as violências contra a pessoa idosa decorrem de um desequilíbrio entre fatores protetores e fatores de risco sociais, comunitários, relacionais e individuais, que interagem, tanto no âmbito social, na dimensão comunitária, no campo das relações mais próximas e no âmbito individual' ${ }^{7}$. As causas da violência no idoso são variadas e muitos fatores afetam sua ocorrência.

Nesse contexto surge a importância da Enfermagem gerontológica através da capacitação profissional no desenvolvimento de uma assistência robusta, qualificada e segura, sendo necessário ressignificar as ações de atenção ao idoso, respeitando a pluralidade e vislumbrando ações de atuação.

\section{O isolamento social e sua relação com a violência contra idosos em tempos de pandemia}

Medidas de restrição social foram adotadas na maioria dos países do mundo com o objetivo de controlar o avanço e a transmissão da infecção causada pela COVID-19, bem como evitar o colapso dos sistemas de saúde público e privado. Contudo, essa estratégia caracteriza-se como um fator de risco para a ocorrência da violência contra a pessoa idosa e da subnotificação dos casos aos órgãos competentes.

$\mathrm{O}$ isolamento social no combate a pandemia da COVID-19 aumentou de forma exponencial pedidos de ajuda de vítimas de violência doméstica. Trata-se de um fenômeno complexo e multifatorial que afeta a 
comunidade, a família e a pessoa. No mundo, estima-se que um entre seis idosos vivenciam algum tipo de violência ${ }^{(1)}$. No Brasil, as denúncias aumentaram em até $50 \%$ durante esse período ${ }^{(8)}$.

A violência doméstica e familiar sofrida pela pessoa idosa geralmente ocorre de forma silenciosa e encoberta pelas relações de proximidade entre a vítima e o autor da agressão. O idoso apresenta dificuldade de manifestar seus sentimentos, pela proximidade que tem com o agressor, vergonha, insegurança, medo de retaliações e abandono, de não ser escutada/o quanto à queixa, assim como a dependência mútua entre o idoso e a família/cuidadores podem agravar ainda mais as situações. As agressões normalmente são praticadas por filho, filhas, cônjuges, netos, netas, irmãos, irmãs ou parentes, vizinhos próximos, pessoas com laços consanguíneos ou conhecidos da vítima ${ }^{(7)}$.

Quando em situação de fragilidade, como ser acamado, cadeirante, apresentar doenças incapacitantes, a exposição à violência é ainda mais desumana. Nesses casos, a depender da gravidade, pode causar danos irreversíveis a vida, de natureza psicológica e/ou física, capaz de provocar lesões graves, inclusive a morte.

$\mathrm{O}$ idoso que sofre de violência possui um risco maior de morte prematura quando comparado àqueles que não sofreram esse tipo de dano durante a vida, visto o impacto físico, psicológico e emocional do ato(7). Por isso, a importância da identificação dos idosos em situação de risco, sinais de violência e a necessidade de adoção de medidas de prevenção e tratamento.

Nesse cenário de isolamento, alguns fatores podem contribuir para a ocorrência da violência contra a pessoa idosa, como o desemprego, diminuição na renda familiar, estresse, sobrecarga de trabalho, ausência de cuidadores formais, uso de álcool e outras drogas, bem como a convivência com o agressor. Esses fatores associados à insipiência de contato com familiares, amigos, serviços de assistência social e outros serviços de saúde podem favorecer a subnotificação dos casos de violência ${ }^{(5)}$.

O preconceito devido à idade conhecido com o ageísmo também é revelado nessa situação. A predição do vírus pelas pessoas mais envelhecidas e a necessidade de manutenção do isolamento social, pode gerar o descontentamento de alguns e o sentimento de raiva pela perda da liberdade do agressor, contribuindo para o aumento nos casos de violência doméstica contra a pessoa idosa ${ }^{(7,9)}$.

As pessoas idosas em geral por medo, não comunicam à violência que está sendo vitimado aos responsáveis, porém é importante estar atentos aos sinais que podem ajudar a identificar casos de violência, principalmente em tempo de pandemia, onde o distanciamento as consultas presenciais aos serviços de saúde tornam-se complexos. Vale observar sinais repentinos de: falta apetite, perda de peso, mudança de humor e de comportamento, higienização precária, presença de equimose, hematomas ou qualquer tipo de ferimento, óculos com armação quebrada, intensificação do isolamento, muito medo ou exagero no respeito a quem está cuidando ou a qualquer pessoa que se relaciona direta ou indiretamente com o idoso.

É importante estar atento também aos sinais de abuso físico que utiliza a força que resulta em danos como: dor e prejuízo físico; o abuso sexual normalmente causando sérios prejuízos para o idoso tanto do ponto de vista físico, emocional e psicológico, contato sexual não consensual; o abuso emocional apresentando uma carga significativa de angústia, sofrimento e dor e por fim, porém, não menos importante os sinais de exploração financeira pelo uso ilegal ou inapropriado dos bens do idoso, gerando insegurança, aflição e medo de não honrar os seus compromissos.

Quanto ao perfil do agressor, estudos ${ }^{(10,11)}$ mostram que o agressor normalmente é um membro da família ou pessoas muito próximas ao idoso, do sexo masculino, adultos/ adulto jovem representado por netos e sobrinhos. Existe uma maior prevalência entre os que são desempregados, alcoolista e ou dependente de outras drogas ilícitas, dependente do idoso financeiramente ou emocionalmente, normalmente apresenta um histórico familiar de violência e reside com o idoso na mesma casa ou próximo. Contudo, não existe nenhuma relação com religião ou outro credo. Normalmente, o agressor inicia pela agressão verbal, seguida da física, motivada geralmente pelo uso abusivo de álcool ou dependência de drogas, bem como sensibilidade emocional.

Por outro lado, a saúde do idoso pode ser afetada, comprometida e até mesmo agravada pelo isolamento social e pela solidão. $O$ receio de exposição ao vírus, ainda pouco conhecido e ameaçador, tanto para as 
pessoas que integram a rede de apoio, como principalmente para os idosos, contribuem ainda mais para o isolamento. Desse modo, pode colaborar para o aumento dos casos de ansiedade, depressão e violência ${ }^{(12)}$.

$O$ isolamento para além da violência, pode também atrapalhar ou impedir o acesso do idoso a benefícios essenciais para o atendimento de suas necessidades básicas e serviços de saúde, os quais podem contribuir para o agravamento das condições crônicas de saúde da pessoa idosa.

Nessa conjuntura, o isolamento social também está associado fundamentalmente ao amparo à população idosa. Porém esse isolamento pode apresentar alguns efeitos não desejados tanto para o idoso que precisa de assistência especializada por profissionais quanto àqueles que precisam de cuidados de seus familiares, aqueles que residem em instituições de longa permanência, como para os cuidadores profissionais ou não. Infere-se que entre os idosos é comum a existência de doenças crônicas, como: hipertensão, doença pulmonar, diabetes melitus, asma, cardiopatias, tornando dessa forma o idoso ainda mais vulnerável às complicações das doenças.

Desse modo, os profissionais de saúde, em especial o enfermeiro, em parceria com os órgãos competentes de proteção ao idoso, precisam usar de ferramentas capazes de identificar sinais de vitimização e violência ao idoso, bem como as características comportamentais do agressor a fim de traçar estratégias capazes de minimizar a ocorrência do ato.

\section{Estratégias de redução da violência em tempos de pandemia}

Na redução da violência a pessoa idosa em tempos de pandemia, a enfermagem gerontológica deverá traçar ações direcionadas ao idoso, cuidadores, outras pessoas da sua convivência e ao contexto os quais estão inseridos. Para isso, é preciso atuar por meio da capacitação profissional para o uso de tecnologias que ajudem no desenvolvimento de uma assistência robusta, qualificada e segura, sendo necessário ressignificar as ações de atenção ao idoso, respeitando a pluralidade e vislumbrando ações para o presente e, principalmente para o futuro.

Nesse sentido, o estabelecimento de intervenções para minimização de violência contra o idoso neste contexto atual torna-se primordial para redução de riscos e preservação da qualidade de vida desta população, dentre as quais destaca-se a rede de apoio e fomento a pesquisas que envolvam o cuidador e a pessoa idosa.

A ampliação da rede de apoio é fundamental para o combate à violência contra a pessoa idosa e como estratégia de enfrentamento neste momento de pandemia cita-se as que permitem o acesso não presencial, com atendimentos virtuais. A utilização de canais telefônicos constitui uma especial intervenção para a proteção dos idosos e cuidadores ${ }^{(7,8,12)}$.

O contato interpessoal limitado faz com que os profissionais de saúde busquem ferramentas tecnológicas para acompanhar esses indivíduos. A vídeo chamada é uma alternativa que se mostra promissora para observar os idosos em seu ambiente doméstico, sendo possível avaliar como estão vivendo, cuidando de si mesmo e sendo cuidado pelos outros. Por outro lado, também é possível avaliar o estresse do cuidador, a capacidade de manutenção do cuidado ao idoso e o acesso aos recursos e suprimentos necessários, como os medicamentos. Desse modo, mesmo com a distância, busca-se identificar e proteger o idoso da violência doméstica, com o possível direcionamento aos órgãos competentes ${ }^{(5)}$.

Salienta-se a necessidade dessas ferramentas virtuais, pois oferecem suporte adequado e pautado nas peculiaridades desta população. A ampliação deste serviço com o suporte de equipes multidisciplinares ${ }^{(12)}$, devem constar no planejamento de implementação das políticas públicas relacionadas à pessoa idosa, expandindo os serviços e coordenando os já existentes para atender as necessidades emergentes da pandemia.

O isolamento social ocasionado pela pandemia contribui para o tensionamento nas relações, em especial dos cuidadores, pois ocorre um aumento da sobrecarga de atividades e consequentemente do estresse. Alguns fatores funcionam como gatilho e contribuem para este processo, dentre os quais cita-se: dificuldades de cuidar de si, medo de transmitir a doença para o idoso ou adoecer ${ }^{(7)}$. 
É primordial a discussão de estratégias de apoio aos cuidadores, pois é necessário cuidar de quem cuida. O desenvolvimento de programas virtuais de apoio aos cuidadores com desenvolvimento de atividades relacionadas ao autocuidado com estímulo da adoção de hábitos que enfatizem a qualidade de vida, é uma alternativa para minimização do estresse.

Ratifica-se a necessidade de investimento em pesquisas que envolvam os cuidadores nesse período da pandemia ${ }^{(12)}$. Existe uma necessidade de investigação sobre o estresse nesse público, bem como a identificação dos principais fatores para sobrecarga nesse contexto atual. Acrescentar nos questionários disponibilizados para os cuidadores, perguntas relacionadas à violência contra a pessoa idosa também contribuem para o levantamento das principais causas que levam à violência. Conhecendo essas nuances, será possível a adoção de medidas plausíveis para amparo deste grupo, impactando no cuidado mais assertivo à pessoa idosa.

É premente a necessidade de atenção aos desafios impostos pelo isolamento social nas relações sociais/ familiares que envolvem a pessoa idosa. Conhecer os fatores motivados dos atos de violência é fundamental para o direcionamento de estratégias assertivas. Atentando para as necessidades de saúde mental de todos os envolvidos, bem como, fornecendo subsídios para a convivência sem e/ou minimização violência durante a crise ocasionada pela pandemia.

\section{CONSIDERAÇÕES FINAIS}

O panorama apresentado quanto à violência relacionada à pandemia da COVID-19, pode agravar o risco de violência contra o idoso. Agravando o abandono afetivo com o distanciamento social, o idoso passa a receber menos ou nenhuma visita; existência anterior de histórico de violência na família, dessa forma há uma maior chance de recorrência; sensação intensa de medo e insegurança por fazer parte ao grupo de maior vulnerabilidade à COVID-19 pela severidade do quadro clínico e a maior letalidade; Cansaço e estresse dos familiares, por causa do trabalho excessivo; problemas financeiros e sobrecarga emocional, relacionado ao quadro de dependência total ou parcial do idoso. É importante a adoção de estratégias que, previnam, minimizem e cuidem dessa população vitimizada, tendo a inserção da enfermagem em todas as etapas.

\section{AGRADECIMENTO}

Ao Departamento Científico de Enfermagem Gerontológica da ABEn Nacional.

\section{REFERÊNCIAS}

1. World Health Organization. WHO Timeline - COVID-19, 2020. Disponível em: https://www.who.int/news-room/ detail/27-04-2020-who-timeline---covid-19

2. Johns Hopkins University \& Medical. Coronavirus Resource Center. 2020. Disponível em: https://coronavirus.jhu.edu/map.html

3. Rothe C, Schunk M, Sothmann P, Bretzel G, Froeschl G, Wallrauch C, Zimmer T, Thiel V, Janke C, Guggemos W, Seilmaier M, Drosten C, Vollmar P, Zwirglmaier K, Zange S, Wölfel R, Hoelscher M. Transmission of 2019-nCoV Infection from an Asymptomatic Contact in Germany. N Engl J Med. 2020 Mar 5; 382(10):970-971.

4. Santini Z, Jose P, Cornwell E, et al. Social disconnectedness, perceived isolation, and symptoms of depression and anxiety among older Americans (NSHAP): a longitudinal mediation analysis. Lancet Public Health. 2020; 5: e62-e70

5. Makaroun LK, Bachrach RL, Rosland AM. Elder abuse in the time of COVI-19 - increased risk for older adults and their caregivers. The American Journal of Geriatric Psychiatry (2020), doi: https://doi.org/10.1016/j.jagp.2020.05.017

6. Souza ERD, Minayo MCDS. The insertion of the vilence against elderly theme at health care public policies in Brazil. Ciênc saúde coletiva [Internet] 2010 [cited 2016 Nov 21];15(6):2659-2668. Available from: http://www.scielo.br/pdf/csc/v15n6/ a02v15n6.pdf 
7. Campbel AM. An Increasing Risk of Family Violence during the Covid-19 Pandemic: Strengthening Community Collaborations to Save Lives. Forensic Science International: Reports, 2020.

8. Ribeiro, AP., et al. O que fazer para cuidar das pessoas idosas e evitar a violência em época da pandemia?. Site da Associação Brasileira de Saúde Coletiva - ABRASCO, 2020. Disponível em: https://www.abrasco.org.br/site/ noticias/o-que-fazer-para-cuidar-das-pessoas-idosas-e-evitar-as-violencias-em-epoca-de-pandemia-artigo/48196/

9. Han, SD, Mosqueda L. Elder abuse in the COVID 19 era. J Am Geriatr Soc., 2020, doi: https://10.1111/jgs.16496

10. Matos NM., et al. Profile of aggressors of older adults receiving care at a geriatrics and gerontology reference center in the Distrito Federal (Federal District), Brazil. Rev. Bras. Geriatr. Gerontol, 2019;22(5): e190095. doi: https://doi. org/10.1590/1981-22562019022.190095

11. Rodrigues, RAP, et al., Older adults in three Brazilian cities. Rev Bras Enferm. 2017;70(4):783-91. doi: http://dx.doi. org/10.1590/0034-7167-2017-0114

12. Elman, A, et al. Effects of the COVID-19 Outbreak on Elder Mistreatment and Response in New York City: Initial Lessons. Journal of Applied Gerontology, 2020, doi: https://10.1177/0733464820924853. 